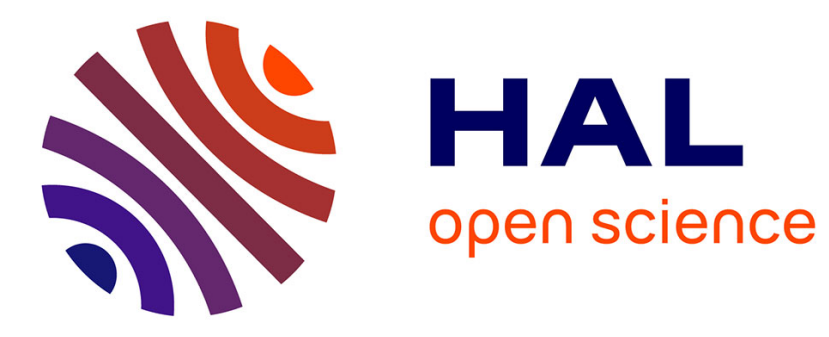

\title{
Bearing rigidity maintenance for formations of quadrotor UAVs
}

Fabrizio Schiano, Paolo Robuffo Giordano

\section{To cite this version:}

Fabrizio Schiano, Paolo Robuffo Giordano. Bearing rigidity maintenance for formations of quadrotor UAVs. ICRA 2017 - IEEE International Conference on Robotics and Automation, May 2017, Singapore, Singapore. pp.1467 - 1474, 10.1109/ICRA.2017.7989175 . hal-01482422v2

\section{HAL Id: hal-01482422 \\ https://hal.inria.fr/hal-01482422v2}

Submitted on 13 Aug 2017

HAL is a multi-disciplinary open access archive for the deposit and dissemination of scientific research documents, whether they are published or not. The documents may come from teaching and research institutions in France or abroad, or from public or private research centers.
L'archive ouverte pluridisciplinaire HAL, est destinée au dépôt et à la diffusion de documents scientifiques de niveau recherche, publiés ou non, émanant des établissements d'enseignement et de recherche français ou étrangers, des laboratoires publics ou privés. 


\title{
Bearing Rigidity Maintenance for Formations of Quadrotor UAVs
}

\author{
Fabrizio Schiano and Paolo Robuffo Giordano
}

\begin{abstract}
This paper considers the problem of controlling a formation of quadrotor UAVs equipped with onboard cameras with the goal of maintaining bearing rigidity during motion despite the presence of several sensing constraints, that is, minimum/maximum range, limited camera field of view, and possible occlusions caused by the agents of the formation. To this end, a decentralized gradient-based control action is developed, based on a suitable 'degree of infinitesimal rigidity' linked to the spectral properties of the bearing rigidity matrix. The approach is then experimentally validated with five quadrotor UAVs.
\end{abstract}

\section{INTRODUCTION}

Research on multi-robot systems has flourished over the last decades with a number of theoretical and experimental results also made possible by the constant technological advancements in onboard sensing, communication and computing power [1]-[5]. A scenario that still motivates considerable research efforts is that of decentralized formation control of multiple mobile robots based on only local (onboard) sensing and communication, with the aim of deploying highly autonomous robot teams in 'non-trivial' environments (e.g., inside buildings, underwater, underground, or even in deep space) where centralized measuring/communication facilities (such as GPS) are not available.

The use of local sensing (such as onboard cameras or range sensors), however, entails a number of challenges. For instance, in absence of centralized aids, each robot is typically only able to collect measurements and impose control actions in its local body-frame: when exchanging information over communication, the group then faces the need of, e.g., agreeing over some common shared frame where to express any quantity of interest. Furthermore, local (onboard) sensing also forces to cope with any sensing limitation (such as limited range/field of view, or occluded visibility) that can prevent retrieving the needed measurements w.r.t. neighboring robots.

In all these cases, the correct theoretical framework for analyzing and controlling the geometrical properties of robot formations defined in terms of relative measurements has proven to be the theory of formation rigidity [6]. Indeed, rigidity plays the role of a necessary condition for allowing convergence of formation control schemes [7]-[12] and of their dual problem of cooperative localization in a common frame [13]-[17] from the available (body-frame and relative) measurements.

F. Schiano is with Inria at Irisa Rennes, Campus de Beaulieu, 35042 Rennes Cedex, France fabrizio.schiano@inria.fr

P. Robuffo Giordano is with CNRS at Irisa and Inria Rennes, Campus de Beaulieu, 35042 Rennes Cedex, France prgeirisa.fr.

This research was supported by the ANR, Project ANR-14-CE27-0007 SenseFly.
While a number of rigidity-based formation control/localization schemes has been proposed over the years, to the best of our knowledge only a few previous works (e.g., [11]) have considered the issue of maintaining/preserving rigidity of the formation (which is a global property like graph connectivity) during the agent motion despite the possible presence of sensing constraints. This is a fundamental problem since, as explained, losing formation rigidity ultimately prevents convergence of any formation control/localization scheme run by the robot group.

In this respect, this paper considers the problem of bearing rigidity maintenance for a robot group equipped with onboard cameras able to measure relative bearings w.r.t. other robots in visibility. Indeed, cameras are a widespread sensor modality for mobile robots, and the problem of coordinating the motion/formation of a robot group from only camera (bearing) measurements has attracted large attention in the robotics and control community [8], [12], [16], [18]-[21]. Cameras, however, also suffer from all the shortcomings listed above: they only provide relative (and unscaled) measurements in the local body-frame of the measuring agent, and are affected by several sensing constraints such as limited field of view, limited range, and occluded visibility.

Taking inspiration from [9] in this work we then propose a control strategy able to ensure maintenance at all times of a minimum level of bearing rigidity for the robot formation during motion. The controller consists of a decentralized gradient descent action based on a suitable "degree of bearing rigidity" which is directly related to the spectral properties of the so-called bearing rigidity matrix [10]. Furthermore, we assume a directed measurement topology (no need of reciprocal measurements among neighboring pairs), and explicitly consider three typical sensing constraints affecting onboard cameras: $(C 1)$ minimum/maximum range, $(C 2)$ limited field of view (fov), and $(C 3)$ possible occluded visibility because of the alignment of multiple robots in the camera fov. Finally, we experimentally validate the proposed machinery with five quadrotor UAVs navigating in an indoor room. To the best of our knowledge, the problem of bearing rigidity maintenance and the use of the complex (but quite realistic) sensing model $(C 1)-(C 3)$ for camera-based formation control purposes are two novel contributions of this work.

The rest of the paper is organized as follows: Sect. II reviews the modeling assumptions and quickly recaps the main notions of bearing rigidity and related concepts. Then Sect. III presents the various details of the proposed bearing rigidity maintenance strategy together with a discussion on the issue of decentralization and scalability. Subsequently, Sect. IV reports the results of our experimental validation with five quadrotor UAVs, and Sect. V concludes the paper 
and discusses some future directions.

\section{PRELIMINARIES}

\section{A. Agent Model}

Let $\mathcal{W}:\left\{\boldsymbol{O}_{\mathcal{W}}, \boldsymbol{X}_{\mathcal{W}}, \boldsymbol{Y}_{\mathcal{W}}, \boldsymbol{Z}_{\mathcal{W}}\right\}$ represent a world frame with $Z_{\mathcal{W}}$ aligned with the vertical (gravity) direction. Following the modeling assumptions of [10], [11], [14], [17], [18], in this work we consider a group of $N$ 'velocitycontrolled' quadrotor UAVs with agent dynamics

$$
\left(\begin{array}{c}
\dot{\boldsymbol{p}}_{i} \\
\dot{\psi}_{i}
\end{array}\right)=\left(\begin{array}{cc}
\boldsymbol{R}_{i} & 0 \\
\mathbf{0} & 1
\end{array}\right)\left(\begin{array}{c}
\boldsymbol{u}_{i} \\
w_{i}
\end{array}\right)
$$

where $\boldsymbol{p}_{i} \in \mathbb{R}^{3}$ is the quadrotor $3 \mathrm{D}$ position in $\mathcal{W}, \psi_{i} \in \mathcal{S}^{1}$ the yaw angle about $\boldsymbol{Z}_{\mathcal{W}}$, and $\boldsymbol{R}_{i}=\boldsymbol{R}_{z}\left(\psi_{i}\right) \in S O(3)$ the associated rotation matrix. The quantities $\boldsymbol{u}_{i} \in \mathbb{R}^{3}$ and $w_{i} \in \mathbb{R}$ represent the body-frame linear velocity and yaw rate which are assumed to be known and controllable ${ }^{1}$. The configuration of the $i$-th quadrotor is then denoted with $\boldsymbol{q}_{i}=\left(\boldsymbol{p}_{i}, \psi_{i}\right) \in \mathbb{R}^{3} \times \mathcal{S}^{1}$, while $\boldsymbol{q}=(\boldsymbol{p}, \boldsymbol{\psi}) \in\left(\mathbb{R}^{3} \times \mathcal{S}^{1}\right)^{N}$ is the configuration of the whole formation, with $\boldsymbol{p}=$ $\left(\boldsymbol{p}_{1}, \ldots \boldsymbol{p}_{N}\right) \in \mathbb{R}^{3 N}$ and $\boldsymbol{\psi}=\left(\psi_{1}, \ldots \psi_{N}\right) \in \mathcal{S}^{N}$.

Each quadrotor is also assumed equipped with an onboard calibrated camera that allows an agent $i$ to measure the relative bearing vector w.r.t. an agent $j$ in visibility, i.e., the $3 \mathrm{D}$ unit-norm vector

$$
\boldsymbol{\beta}_{i j}=\boldsymbol{R}_{i}^{T} \frac{\boldsymbol{p}_{j}-\boldsymbol{p}_{i}}{\left\|\boldsymbol{p}_{j}-\boldsymbol{p}_{i}\right\|}=\boldsymbol{R}_{i}^{T} \frac{\boldsymbol{p}_{i j}}{d_{i j}} \in \mathcal{S}^{2},
$$

where $\boldsymbol{p}_{i j}=\boldsymbol{p}_{j}-\boldsymbol{p}_{i}$ and $d_{i j}=\left\|\boldsymbol{p}_{j}-\boldsymbol{p}_{i}\right\|$. As explained in [18], vector $\boldsymbol{\beta}_{i j}$ can be directly retrieved by derotating the actual bearing measurement (from the onboard camera of robot $i$ ) by the known orientation w.r.t. $Z_{\mathcal{W}}$ (provided by the onboard IMU of robot $i$ ).

\section{B. Directed Bearing Rigidity in $\mathbb{R}^{3} \times \mathcal{S}^{1}$}

We now quickly recap the main definitions and properties of directed bearing formations and bearing rigidity in $\mathbb{R}^{3} \times \mathcal{S}^{1}$ that are relevant to this work. The interested reader is referred to [10], [11], [14] for all the details.

Let $\mathcal{G}=(\mathcal{V}, \mathcal{E})$ be a directed (sensing) graph, where $\mathcal{V}=\{1 \ldots N\}$ is the vertex set and $\mathcal{E} \subseteq \mathcal{V} \times \mathcal{V}$ the edge set. We let $\mathcal{N}_{i}=\{j \in \mathcal{V} \mid(i, j) \in \mathcal{E}\}$ represent the usual set of neighbors of an agent $i$, and $\mathcal{O}_{i}=\{j \in \mathcal{V} \mid(j, i) \in \mathcal{E}\}$ the set of agents $j$ for which $i$ is a neighbor ${ }^{2}$. We also let $\mathbf{1}_{N}$ and $\boldsymbol{I}_{N}$ represent a vector of all ones and the identity matrix of dimension $N$, respectively. Presence of an edge $e_{k}=(i, j)$ in $\mathcal{E}$ represents the possibility for agent $i$ to measure the relative bearing $\boldsymbol{\beta}_{i j}$ (2) w.r.t. agent $j$. Graph $\mathcal{G}$ is designed as directed as we do not require, in general, reciprocity of the relative bearing measurements. We assume, however, that agents $i$ and $j$ can communicate if either $(i, j) \in \mathcal{E}$ or

\footnotetext{
${ }^{1}$ We stress that, as in [10], [11], [14], [17], [18], the (absolute) yaw angle $\psi_{i}$ is not considered as an available quantity to the $i$-th quadrotor. As a consequence, the $N$ quadrotors are not assumed to share, as a group, a common (global) reference frame where to express local measurements and control inputs.

${ }^{2}$ This distinction between sets $\mathcal{N}_{i}$ and $\mathcal{O}_{i}$, needed because of the directed nature of graph $\mathcal{G}$, will play an important role in Sects. III-B-III-C.
}

$(j, i) \in \mathcal{E}$ (i.e., the communication graph is taken as the undirected counterpart of the directed sensing graph $\mathcal{G}$ ).

A framework/formation is the pair $(\mathcal{G}, \boldsymbol{q})$ where $\boldsymbol{p}_{i}: \mathcal{V} \rightarrow$ $\mathbb{R}^{3}$ and $\psi_{i}: \mathcal{V} \rightarrow \mathcal{S}^{1}$ map each vertex in $\mathcal{V}$ to an agent configuration $\boldsymbol{q}_{i}=\left(\boldsymbol{p}_{i}, \psi_{i}\right)$ in $\mathbb{R}^{3} \times \mathcal{S}^{1}$. Given a framework, the associated bearing function is the map $\boldsymbol{\beta}_{\mathcal{G}}(\boldsymbol{q}):\left(\mathbb{R}^{3} \times\right.$ $\left.\mathcal{S}^{1}\right)^{N} \rightarrow\left(\mathcal{S}^{2}\right)^{|\mathcal{E}|}$

$$
\boldsymbol{\beta}_{\mathcal{G}}(\boldsymbol{q})=\left[\boldsymbol{\beta}_{e_{1}}^{T} \ldots \boldsymbol{\beta}_{e_{|\mathcal{E}|}^{T}}^{T}\right]^{T}
$$

where the notation $e_{i} \in \mathcal{E}$ is used to represent a directed edge in the graph $\mathcal{G}$ according to any chosen labeling. The Jacobian of $\boldsymbol{\beta}_{\mathcal{G}}(\boldsymbol{q})$ w.r.t. the configuration vector $\boldsymbol{q}$

$$
\mathcal{B}_{\mathcal{G}}^{\mathcal{W}}(\boldsymbol{q})=\frac{\partial \boldsymbol{\beta}_{\mathcal{G}}(\boldsymbol{q})}{\partial \boldsymbol{q}} \in \mathbb{R}^{3|\mathcal{E}| \times 4 N}
$$

is denoted as the world-frame bearing rigidity matrix. The $(3 \times 4 N) k$-th row block of $\boldsymbol{B}_{\mathcal{G}}^{\mathcal{W}}(\boldsymbol{q})$ associated to an edge $e_{k}=(i, j)$ has expression

$[\mathbf{0}-\underbrace{-\frac{\boldsymbol{P}_{i j} \boldsymbol{R}_{i}^{T}}{d_{i j}}}_{i}-\mathbf{0}-\underbrace{\frac{\boldsymbol{P}_{i j} \boldsymbol{R}_{i}^{T}}{d_{i j}}}_{j}-\mathbf{0}-\underbrace{-\boldsymbol{S}_{i j}}_{3 N+i}-\mathbf{0}-]$

where $\boldsymbol{P}_{i j}=\boldsymbol{I}_{3}-\boldsymbol{\beta}_{i j} \boldsymbol{\beta}_{i j}^{T}$ is the orthogonal projector onto the orthogonal complement of $\boldsymbol{\beta}_{i j}$, and $\left.\boldsymbol{S}=\left[\begin{array}{lll}0 & 0 & 1\end{array}\right]^{T}\right]_{\times}$with $[\cdot]_{\times}$indicating the usual skew-symmetric matrix operator.

The world-frame rigidity matrix relates variations in the bearing function to the world-frame agent velocities $(\dot{\boldsymbol{p}}, \dot{\boldsymbol{\psi}})$. Exploiting (1), one can also define a body-frame bearing rigidity matrix $\mathcal{B}_{\mathcal{G}}(\boldsymbol{q})$ as

$$
\begin{aligned}
\dot{\boldsymbol{\beta}}_{\mathcal{G}} & =\mathcal{B}_{\mathcal{G}}^{\mathcal{W}}(\boldsymbol{q})\left[\begin{array}{cc}
\operatorname{diag}\left(\boldsymbol{R}_{i}\right) & \mathbf{0} \\
\mathbf{0} & \boldsymbol{I}_{N}
\end{array}\right]\left[\begin{array}{c}
\boldsymbol{u} \\
\boldsymbol{w}
\end{array}\right]=\boldsymbol{B}_{\mathcal{G}}^{\mathcal{W}} \boldsymbol{T}(\boldsymbol{\psi})\left[\begin{array}{c}
\boldsymbol{u} \\
\boldsymbol{w}
\end{array}\right] \\
& =\mathcal{B}_{\mathcal{G}}(\boldsymbol{q})\left[\begin{array}{c}
\boldsymbol{u} \\
\boldsymbol{w}
\end{array}\right]
\end{aligned}
$$

which explicits the dependance on the body-frame velocity inputs $\boldsymbol{u}=\left(\boldsymbol{u}_{1}, \ldots, \boldsymbol{u}_{N}\right)$ and $\boldsymbol{w}=\left(w_{1}, \ldots, w_{N}\right)$. The $(3 \times 4 N) k$-th row block of $\mathcal{B}_{\mathcal{G}}(\boldsymbol{q})$ associated to an edge $e_{k}=(i, j)$ has expression

$$
[-\mathbf{0}-\underbrace{-\frac{\boldsymbol{P}_{i j}}{d_{i j}}}_{i}-\mathbf{0}-\underbrace{\frac{\boldsymbol{P}_{i j}{ }^{i} \boldsymbol{R}_{j}}{d_{i j}}}_{j}-\mathbf{0}-\underbrace{-\boldsymbol{S}_{i j}}_{3 N+i}-\mathbf{0}-],
$$

where ${ }^{i} \boldsymbol{R}_{j}=\boldsymbol{R}_{z}\left(\psi_{j}-\psi_{i}\right)=\boldsymbol{R}_{z}\left(\psi_{i j}\right)$. It is worth noting that (6) depends on the (measured) bearing $\boldsymbol{\beta}_{i j}$, on the interdistance $d_{i j}$, and on the relative orientation $\psi_{i j}$ among agents $i$ and $j$ (rather than on the absolute orientations $\psi_{i}$ as in (4)).

Letting $\mathcal{N}(\cdot)$ represent the span of the null-space of a matrix, a framework $(\mathcal{G}, \boldsymbol{q})$ is said to be infinitesimally bearing rigid at some point $\boldsymbol{q}$ if $\mathcal{N}\left(\boldsymbol{B}_{\mathcal{G}}^{\mathcal{W}}(\boldsymbol{q})\right)=\mathcal{N}\left(\boldsymbol{B}_{\mathcal{K}_{N}}^{\mathcal{W}}(\boldsymbol{q})\right)$, with $\mathcal{K}_{N}$ being the complete directed graph. Otherwise a framework is said to be infinitesimally roto-flexible. It can be shown that $\operatorname{dim} \mathcal{N}\left(\mathcal{B}_{\mathcal{K}_{N}}^{\mathcal{W}}(\boldsymbol{q})\right)=5$, see [10]. Therefore, a framework $(\mathcal{G}, \boldsymbol{q})$ in $\mathbb{R}^{3} \times \mathcal{S}^{1}$ is infinitesimally rigid if and only if $\operatorname{rank}\left(\boldsymbol{B}_{\mathcal{G}}^{\mathcal{W}}(\boldsymbol{q})\right)=4 N-5$. The same considerations extend to the body-frame bearing rigidity matrix since 
$\operatorname{rank}\left(\boldsymbol{B}_{\mathcal{G}}(\boldsymbol{q})\right)=\operatorname{rank}\left(\boldsymbol{B}_{\mathcal{G}}^{\mathcal{W}}(\boldsymbol{q})\right)$, being $\boldsymbol{T}(\boldsymbol{\psi})$ in (5) a square non-singular matrix.

\section{Cooperative localization from bearing measurements}

As suggested by (6), the implementation of bearing formation control algorithms for agents evolving in $\mathbb{R}^{3} \times$ $\mathcal{S}^{1}$ requires the availability of the relative orientation ${ }^{i} \boldsymbol{R}_{j}$ and, possibly, distance $d_{i j}$ among neighboring robots (see, e.g., [10], [11], [18]), which, in our context, are not assumed to be directly measurable. A possible workaround is to then estimate these (needed) quantities via some bearing-based cooperative localization scheme.

In this respect, a decentralized localization algorithm for rigid frameworks in $\mathbb{R}^{3} \times \mathcal{S}^{1}$ has been recently presented in [10], [17]. Letting $\hat{\boldsymbol{q}}_{i}=\left(\hat{\boldsymbol{p}}_{i}, \hat{\psi}_{i}\right)$ represent the estimation of the true agent $i$ configuration $\boldsymbol{q}_{i}$, the localization algorithm guarantees that, at convergence,

$$
\left\{\begin{aligned}
\hat{\boldsymbol{p}} & =\left(\boldsymbol{I}_{N} \otimes \boldsymbol{R}(\bar{\psi})\right) \boldsymbol{p}+\mathbf{1}_{N} \otimes \boldsymbol{t} \\
\hat{\boldsymbol{\psi}} & =\boldsymbol{\psi}+\mathbf{1}_{N} \bar{\psi}
\end{aligned}\right.
$$

for an arbitrary translation $t \in \mathbb{R}^{3}$ and rotation angle $\bar{\psi} \in \mathcal{S}^{1}$ (with $\otimes$ representing the matrix Kronecker product). Any two neighboring agents can then exchange their estimated poses $\hat{\boldsymbol{q}}_{i}$ and $\hat{\boldsymbol{q}}_{j}$ over local communication for then replacing the unknown $d_{i j}$ and $\psi_{i j}$ with the estimated $\hat{d}_{i j}=\left\|\hat{\boldsymbol{p}}_{i}-\hat{\boldsymbol{p}}_{j}\right\|$ and $\hat{\psi}_{i j}=\hat{\psi}_{j}-\hat{\psi}_{i}$. The localization algorithm presented in [10], [17] is fully decentralized and can cope with any time-varying bearing function $\boldsymbol{\beta}_{\mathcal{G}}(\boldsymbol{q}(t))$ (i.e., the agents do not need to remain stationary or move in 'special' ways) as long as the formation is infinitesimally bearing rigid. It does, however, require availability of at least one interagent distance $d_{\iota \kappa}$ among an arbitrary agent pair $(\iota, \kappa)$ for fixing the scale of the formation (which, otherwise, would be unobservable from only bearing measurements). This (possibly single) inter-agent distance can be either considered as an additional measurement among a special agent pair equipped with a distance sensor [10], or it can be estimated online by processing the measured bearings and the known agent body-frame velocities $\left(\boldsymbol{u}_{i}, w_{i}\right)$ [17] (thus, without requiring in this case any additional sensing capability).

We will then assume, from now on, that the localization algorithm [10], [17] is run by the agent group, and will treat $\hat{\boldsymbol{q}}_{i}$ as a sufficiently good approximation of the true $\boldsymbol{q}_{i}$ in the sense of (7).

\section{A BEARING RIGIDITY MAINTENANCE STRATEGY}

The goal of this section is to present an algorithm for bearing rigidity maintenance able to also take into account some typical limitations of the employed onboard sensors (cameras), that is, minimum/maximum range, limited field of view, and possible line-of-sight (visibility) occlusions due to the alignments of multiple robots in the camera fov.

Our strategy is inspired by the connectivity/(distance) rigidity maintenance controllers presented in [3], [9], [22] and can be summarized as follows: the sensing constraints affecting a pair of agents $(i, j)$ are encoded in a suitable

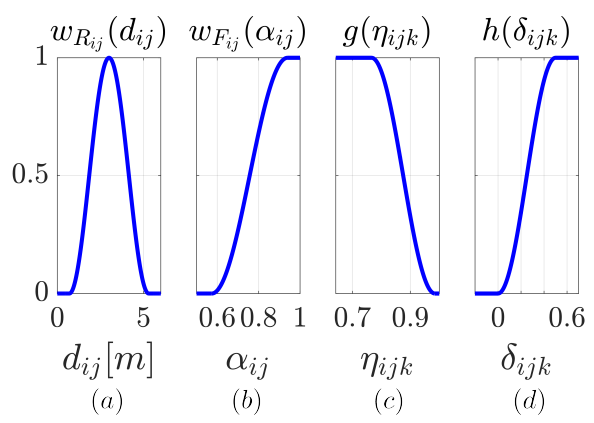

Fig. 1: Representative shapes for the weights $w_{R_{i j}}\left(d_{i j}\right)$ (a), $w_{F_{i j}}\left(\alpha_{i j}\right)(\mathrm{b}), g\left(\eta_{i j k}\right)(\mathrm{c})$, and $h\left(\delta_{i j k}\right)(\mathrm{d})$

scalar weight ${ }^{3} w_{i j}=w_{k}$ associated to the edge $e_{k}=(i, j)$, with $w_{k}=0$ if the constraint is not satisfied, and $0<$ $w_{k} \leq 1$ otherwise ${ }^{4}$. Let $\boldsymbol{W}=\operatorname{diag}\left(w_{k} \boldsymbol{I}_{3}\right) \in \mathbb{R}^{3|\mathcal{E}| \times 3|\mathcal{E}|}$ be a diagonal matrix collecting all the $|\mathcal{E}|$ weights $w_{k}=$ $w_{i j}$, and define the weighted body-frame rigidity matrix as $\boldsymbol{W} \mathcal{B}_{\mathcal{G}}=\boldsymbol{W} \mathcal{B}_{\mathcal{G}}^{\mathcal{W}} \boldsymbol{T}$. Any weight $w_{k} \rightarrow 0$ will cause the corresponding $k$-th row block of $\boldsymbol{W} \boldsymbol{B}_{\mathcal{G}}$, associated to edge $e_{k}=(i, j)$, to vanish, potentially diminishing the rank of $\boldsymbol{W} \mathcal{B}_{\mathcal{G}}$ (and, thus, possibly threatening bearing rigidity of the framework). The agents will then implement a gradientbased controller able to preserve infinitesimal bearing rigidity of the formation by ensuring fulfilment of the rank condition $\operatorname{rank}\left(\boldsymbol{W} \mathcal{B}_{\mathcal{G}}^{\mathcal{W}}\right)=4 N-5$ for the weighted bearing rigidity matrix. The next sections will detail the various steps needed to implement this gradient control action.

\section{A. Design of the inter-agent weights}

We start addressing the design of the inter-agent weights $w_{i j}$ associated to each edge $e_{k}=(i, j)$ that will encode the sensing constraints among agent pairs. Since, as explained in Sect. I, in this work we consider the three constraints $(C 1)$ minimum/maximum range, $(C 2)$ limited field of view and $(C 3)$ occluded visibility, the weights $w_{i j}$ will then be designed as the product of three individual terms

$$
w_{i j}=w_{R_{i j}} w_{F_{i j}} w_{V_{i j}}
$$

accounting for $(C 1)-(C 3)$, respectively.

1) Minimum/maximum range: As first constraint, we consider that the employed cameras have a minimum/maximum range $d_{\min }<d_{\max }$ beyond which no detection of neighboring quadrotors is possible. Weight $w_{R_{i j}}\left(d_{i j}\right)$ is then designed as a function that smoothly vanishes (with vanishing derivative) when $d_{i j} \rightarrow d_{\min }$ and $d_{i j} \rightarrow d_{\max }$, and has a maximum at the midpoint between $d_{\min }$ and $d_{\max }$. To this end, we choose the following function

$w_{R_{i j}}\left(d_{i j}\right)=\left\{\begin{array}{cc}0 & d_{i j}<d_{\min } \\ \frac{1}{2}+\frac{1}{2} \cos \left(\pi \frac{2 d_{i j}-\left(d_{\min }+d_{\max }\right)}{d_{\max }-d_{\min }}\right) & d_{\min } \leq d_{i j} \leq d_{\max } \\ 0 & d_{i j}>d_{\max }\end{array}\right.$

for which a representative shape is shown in Fig. 1a.

\footnotetext{
${ }^{3}$ With a small abuse of notation, we will use either $w_{k}$ or $w_{i j}$ to indicate the weight associated to an edge $e_{k}=(i, j)$.

${ }^{4} \mathrm{We}$ also note that the introduction of time-varying weights $w_{i j}(t)$ naturally induces a corresponding time-varying edge set $\mathcal{E}(t)$ (the set of all edges $e_{k}$ for which $w_{k}(t)>0$ ).
} 


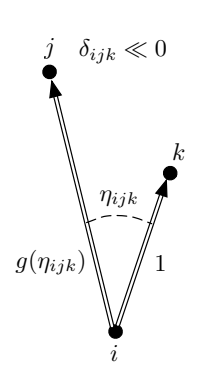

(a)

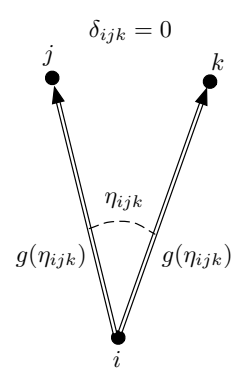

(b)

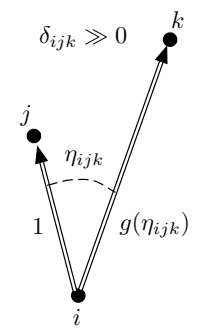

(c)
Fig. 2: Scheme which depicts the weights associated to the occluded visibility between a triple of agents.

2) Limited field of view: A second constraint is related to the limited fov of the onboard cameras. Let $\boldsymbol{o}_{C} \in \mathcal{S}^{2}$ be the (constant and known) direction of the camera optical axis in the quadrotor body-frame and consider the scalar product $\alpha_{i j}=\boldsymbol{o}_{C}^{T} \boldsymbol{\beta}_{i j}$. Clearly, $\alpha_{i j}=1$ when $\boldsymbol{\beta}_{i j}$ and $\boldsymbol{o}_{C}$ are perfectly aligned, and $\alpha_{i j} \rightarrow-1$ as the angle between $\boldsymbol{\beta}_{i j}$ and $\boldsymbol{o}_{C}$ increases. Letting $-1 \leq \alpha_{\min }<1$ represent the camera fov, we then design weight $w_{F_{i j}}$ as the following function with a maximum for $\alpha_{\max } \leq \alpha_{i j} \leq 1$ and smoothly vanishing with vanishing derivative for $\alpha_{i j} \rightarrow \alpha_{\text {min }}$

$$
w_{F_{i j}}\left(\alpha_{i j}\right)=\left\{\begin{array}{cc}
0 & \alpha_{i j}<\alpha_{\min } \\
\frac{1}{2}-\frac{1}{2} \cos \left(\pi \frac{\alpha_{i j}-\alpha_{\min }}{\alpha_{\max }-\alpha_{\min }}\right) & \alpha_{\min } \leq \alpha_{i j} \leq \alpha_{\max } \\
1 & \alpha_{i j}>\alpha_{\max }
\end{array}\right.
$$

A representative shape is shown in Fig. 1b.

3) Occluded visibility: A final constraint considered in this work is that of possible occluded visibility because of two (or more) quadrotors (close to be) aligned in front of a camera (with, thus, the closest quadrotor occluding the visibility of the farthest ones). The design of the weight $w_{V_{i j}}$ meant to encode this constraint requires some care and, thus, we will proceed by steps.

Let us first focus on the situation depicted in Figs. 2(ac) in which an agent $i$ is measuring exactly two agents $j$ and $k$. Our goal is to define a function $w_{V_{i j k}}$ that captures the possible occlusions of agent $k$ (third subscript) on the edge $(i, j)$ (first two subscripts), and, analogously, a function $w_{V_{i k j}}$ capturing the occlusions of $j$ on the edge $(i, k)$. To this end, let $\eta_{i j k}=\boldsymbol{\beta}_{i j}^{T} \boldsymbol{\beta}_{i k}$ represent the (cosine of the) angle between the two bearing measurements of $j$ and $k$ by agent $i$, and $\delta_{i j k}=d_{i k}-d_{i j}$ the relative distance of $k$ and $j$ w.r.t. $i$. Note that $\eta_{i j k}=\eta_{i k j}$ and $\delta_{i j k}=-\delta_{i k j}$.

Since, unlike the previous case, we now wish to penalize small angular displacements between $j$ and $k$ w.r.t. $i$ (which could lead to a possible occlusion), we introduce the following penalty function

$g\left(\eta_{i j k}\right)=\left\{\begin{array}{cc}0 & \eta_{i j k} \geq \eta_{\max } \\ \frac{1}{2}-\frac{1}{2} \cos \left(\pi \frac{\eta_{i j k}-\eta_{\max }}{\eta_{\max }-\eta_{\min }}\right) & \eta_{\min } \leq \eta_{i j k} \leq \eta_{\max } \\ 1 & \eta_{i j k} \leq \eta_{\min }\end{array}\right.$

where $-1 \leq \eta_{\min }<\eta_{\max } \leq 1$ are suitable parameters representing the activation/deactivation of function $g(\cdot)$. Therefore, $g\left(\eta_{i j k}\right)=0$ if agents $j$ and $k$ are close to be aligned from the vantage point of $i\left(\eta_{i j k} \geq \eta_{\max }\right)$, while $g\left(\eta_{i j k}\right)=1$ if agents $j$ and $k$ are far from being aligned $\left(\eta_{i j k} \leq \eta_{\min }\right)$. Figure 1c shows a representative plot of function $g\left(\eta_{i j k}\right)$.

The weight $w_{V_{i j k}}$, associated to the edge $(i, j)$, should be clearly based on the 'angular penalty' function $g\left(\eta_{i j k}\right)$. However, weight $w_{V_{i j k}}$ should also take into account the fact that, regardless of the value of $\eta_{i j k}$, agent $j$ can be occluded by agent $k$ only if agent $k$ is front of $j$ (i.e., if $\delta_{i j k}<0$ ), and symmetrically for the weight $w_{V_{i k j}}$ associated to the edge $(k, j)$. As depicted in Fig. 2(a-c), a possibility is to have:

1) $w_{V_{i j k}}=g\left(\eta_{i j k}\right)$ and $w_{V_{i k j}}=1$ if $\delta_{i j k} \ll 0$ : agent $k$ is in front of $j$ and, thus, only the edge $(i, j)$ is penalized by $g\left(\eta_{i j k}\right)$ (Fig. 2(a));

2) $w_{V_{i j k}}=1$ and $w_{V_{i k j}}=g\left(\eta_{i j k}\right)$ if $\delta_{i j k} \gg 0$ : agent $j$ is in front of $k$ and, thus, only the edge $(i, k)$ is penalized by $g\left(\eta_{i j k}\right)$ (Fig. 2(c));

3) $w_{V_{i j k}}=w_{V_{i k j}}=g\left(\eta_{i j k}\right)$ if $\delta_{i j k}=0$ : agents $j$ and $k$ are at the same distance from $i$ and, therefore, the two edges $(i, j)$ and $(i, k)$ are equally penalized by $g\left(\eta_{i j k}\right)$ (Fig. 2(b))

In order to obtain this goal we then introduce a second penalty function

$h\left(\delta_{i j k}\right)=\left\{\begin{array}{cc}0 & \delta_{i j k} \leq 0 \\ \frac{1}{2}+\frac{1}{2} \cos \left(\pi \frac{\delta_{i j k}-\delta_{\max }}{\delta_{\max }}\right) & 0 \leq \delta_{i j k} \leq \delta_{\max } \\ 1 & \delta_{\max } \leq \delta_{i j k}\end{array}\right.$

where $\delta_{\max }>0$ is an activation parameter. Figure 1d shows a representative plot of function $h\left(\delta_{i j k}\right)$. With this choice of $h\left(\delta_{i j k}\right)$, weight $w_{V_{i j k}}$ can then be chosen as

$$
w_{V_{i j k}}\left(\delta_{i j k}, \eta_{i j k}\right)=\left(1-h\left(\delta_{i j k}\right)\right) g\left(\eta_{i j k}\right)+h\left(\delta_{i j k}\right),
$$

and symmetrically for $w_{V_{i k j}}\left(\delta_{i k j}, \eta_{i k j}\right)$. Because of (12), one can easily verify that the expression in (13) correctly realizes the above-mentioned requirements 1)-3) for weights $w_{V_{i j k}}$ and $w_{V_{i k j}}$.

The last step is to generalize this procedure for producing the (cumulative) weight $w_{V_{i j}}$ on the edge $(i, j)$ to be plugged in (8). The previous $w_{V_{i j k}}$ accounts for the occlusions on edge $(i, j)$ by a single specific agent $k \in \mathcal{N}_{i} /\{j\}$. Clearly, if $\left|\mathcal{N}_{i}\right|<2$ then one should have $w_{V_{i j}} \equiv 1$ (no occlusions are possible if agent $i$ has less than two neighbors). If, on the other hand, $\left|\mathcal{N}_{i}\right| \geq 2$ then, in order to take into account all the possible occlusions on edge $(i, j)$ by any neighbor $k \in \mathcal{N}_{i} /\{j\}$, one can just take the product sequence

$$
w_{V_{i j}}=\prod_{k \in \mathcal{N}_{i} /\{j\}} w_{V_{i j k}}\left(\delta_{i j k}, \eta_{i j k}\right) .
$$

\section{$B$. The bearing rigidity eigenvalue}

Having introduced the weighting machinery meant to encode the sensing constraints considered in this work, we now discuss a suitable measure of bearing rigidity that will be exploited by the rigidity maintenance controller. We will first consider the unweighted case $\left(\boldsymbol{W}=\boldsymbol{I}_{3|\mathcal{E}|}\right)$ and then explicitly introduce the weights $w_{i j}$ in the design. 
As discussed, a framework is infinitesimally bearing rigid iff $\operatorname{rank}\left(\boldsymbol{B}_{\mathcal{G}}^{\mathcal{W}}(\boldsymbol{q})\right)=\operatorname{rank}\left(\boldsymbol{B}_{\mathcal{G}}(\boldsymbol{q})\right)=4 N-5$. This rank condition can be translated into an equivalent spectral condition on the eigenvalues of two suitable corresponding matrixes. To this end, define $\boldsymbol{B}_{\mathcal{G}}^{\mathcal{W}}(\boldsymbol{q})=\boldsymbol{B}_{\mathcal{G}}^{\mathcal{W}}(\boldsymbol{q})^{T} \boldsymbol{B}_{\mathcal{G}}^{\mathcal{W}}(\boldsymbol{q})$ and $\boldsymbol{B}_{\mathcal{G}}(\boldsymbol{q})=$ $\boldsymbol{B}_{\mathcal{G}}^{T}(\boldsymbol{q}) \mathcal{B}_{\mathcal{G}}(\boldsymbol{q})$ as the $4 N \times 4 N$ world-frame and body-frame (weighted) symmetric rigidity matrixes, respectively. These two matrixes are similar since from (5) it follows

$$
\boldsymbol{B}_{\mathcal{G}}(\boldsymbol{q})=\boldsymbol{T}^{T}(\boldsymbol{\psi}) \boldsymbol{B}_{\mathcal{G}}^{\mathcal{W}}(\boldsymbol{q}) \boldsymbol{T}(\boldsymbol{\psi})
$$

and $\boldsymbol{T}(\boldsymbol{\psi})$ is orthonormal. Letting $\lambda_{i}(\boldsymbol{A})$ represent the $i$ th smallest eigenvalue of a square symmetric matrix $\boldsymbol{A}$, infinitesimal bearing rigidity then translates into the condition $\lambda_{6}\left(\boldsymbol{B}_{\mathcal{G}}^{\mathcal{W}}(\boldsymbol{q})\right)=\lambda_{6}\left(\boldsymbol{B}_{\mathcal{G}}(\boldsymbol{q})\right)>0$ (where the similarity between $\boldsymbol{B}_{\mathcal{G}}^{\mathcal{W}}(\boldsymbol{q})$ and $\boldsymbol{B}_{\mathcal{G}}(\boldsymbol{q})$ has been used). Let then $\lambda_{6}^{B}(\boldsymbol{q})$ represent the sixth smallest eigenvalue of the world/bodyframe symmetric rigidity matrix: $\lambda_{6}^{B}(\boldsymbol{q})$ is a natural measure of infinitesimal rigidity and will be denoted from now on as the bearing rigidity eigenvalue. The purpose of this section is to detail the main properties and explicit expressions of $\lambda_{6}^{B}(\boldsymbol{q})$ and of its gradient w.r.t. the $i$-th agent configuration $\boldsymbol{q}_{i}$, which will be later used in Sect. III-C.

A first observation is formalized by the following Proposition.

Proposition 1. The bearing ridigity eigenvalue does not depend on the agent orientations $\boldsymbol{\psi}$, i.e., $\lambda_{6}^{B}(\boldsymbol{q})=\lambda_{6}^{B}(\boldsymbol{p})$.

Proof. The proof will exploit the definition $\lambda_{6}^{B}(\boldsymbol{q})=$ $\lambda_{6}\left(\boldsymbol{B}_{\mathcal{G}}^{\mathcal{W}}(\boldsymbol{q})\right)$ based on the world-frame symmetric rigidity matrix. Let $\overline{\boldsymbol{p}}_{i j}=\boldsymbol{p}_{i j} / d_{i j}$ and $\overline{\boldsymbol{P}}_{i j}=\boldsymbol{I}_{n}-\overline{\boldsymbol{p}}_{i j} \overline{\boldsymbol{p}}_{i j}^{T}$. Exploiting (2) and the identity $\boldsymbol{S} \boldsymbol{R}_{i}=\boldsymbol{R}_{i} \boldsymbol{S}$, it follows that $\boldsymbol{P}_{i j} \boldsymbol{R}_{i}^{T}=\left(\boldsymbol{I}_{N}-\boldsymbol{\beta}_{i j} \boldsymbol{\beta}_{i j}^{T}\right) \boldsymbol{R}_{i}^{T}=\boldsymbol{R}_{i}^{T} \overline{\boldsymbol{P}}_{i j}$ and $\boldsymbol{S} \boldsymbol{\beta}_{i j}=$ $\boldsymbol{R}_{i}^{T} \boldsymbol{S} \overline{\boldsymbol{p}}_{i j}$. The $k$-th row block of $\mathcal{B}_{\mathcal{G}}^{\mathcal{W}}(\boldsymbol{q})$ in (4) associated to an edge $e_{k}=(i, j)$ can then be factorized as

$$
\begin{aligned}
& \boldsymbol{R}_{i}^{T}\left(\psi_{i}\right)[-\mathbf{0}-\underbrace{-\frac{\overline{\boldsymbol{P}}_{i j}}{d_{i j}}}_{i}-\mathbf{0}-\underbrace{\frac{\overline{\boldsymbol{P}}_{i j}}{d_{i j}}}_{j}-\mathbf{0}-\underbrace{-\boldsymbol{S} \overline{\boldsymbol{p}}_{i j}}_{3 N+i}-\mathbf{0}-] \\
& =\boldsymbol{R}_{i}^{T}\left(\psi_{i}\right) \overline{\boldsymbol{B}}_{k}\left(\boldsymbol{p}_{i j}\right),
\end{aligned}
$$

where $\overline{\boldsymbol{B}}_{k}\left(\boldsymbol{p}_{i j}\right) \in \mathbb{R}^{3 \times 4 N}$ does not depend on $\boldsymbol{\psi}$. By stacking all the $\overline{\boldsymbol{B}}_{k}$ into $\overline{\boldsymbol{B}}=\left(\overline{\boldsymbol{B}}_{1}^{T} \ldots \overline{\boldsymbol{B}}_{k}^{T}\right)^{T} \in \mathbb{R}^{3|\mathcal{E}| \times 4 N}$, it follows that the world-frame symmetric rigidity matrix can be actually rewritten as

$$
\boldsymbol{B}_{\mathcal{G}}^{\mathcal{W}}(\boldsymbol{q})=\mathcal{B}_{\mathcal{G}}^{\mathcal{W}}(\boldsymbol{q})^{T} \boldsymbol{B}_{\mathcal{G}}^{\mathcal{W}}(\boldsymbol{q})=\overline{\boldsymbol{B}}(\boldsymbol{p})^{T} \overline{\boldsymbol{B}}(\boldsymbol{p}) .
$$

This shows that $\boldsymbol{B}_{\mathcal{G}}^{\mathcal{W}}(\boldsymbol{q})=\boldsymbol{B}_{\mathcal{G}}^{\mathcal{W}}(\boldsymbol{p})$ and, as a consequence, that $\lambda_{6}^{B}(\boldsymbol{q})=\lambda_{6}^{B}(\boldsymbol{p})$, thus concluding the proof.

We now provide two explicit expressions of $\lambda_{6}^{B}(\boldsymbol{p})$ that will be instrumental in the following developments. Let $\boldsymbol{v}_{6} \in \mathbb{R}^{4 N}$ represent the world-frame normalized eigenvector associated to $\lambda_{6}^{B}$, i.e., such that (using (17)) $\lambda_{6}^{B}=$ $\boldsymbol{v}_{6}^{T} \boldsymbol{B}_{\mathcal{G}}^{\mathcal{W}} \boldsymbol{v}_{6}=\boldsymbol{v}_{6}^{T} \bar{B}^{T} \overline{\boldsymbol{B}} \boldsymbol{v}_{6}$. Consider the partition $\boldsymbol{v}_{6}=$ $\left[\boldsymbol{v}_{p_{1}}^{T} \ldots \boldsymbol{v}_{p_{N}}^{T} v_{\psi_{1}} \ldots v_{\psi_{N}}\right]^{T}$, where $\boldsymbol{v}_{p_{i}} \in \mathbb{R}^{3}$ and $\boldsymbol{v}_{\psi_{i}} \in \mathbb{R}$ are the eigenvector components associated to the position and orientation of agent $i$. By exploiting (16), the properties
$\overline{\boldsymbol{P}}_{i j} \overline{\boldsymbol{P}}_{i j}=\overline{\boldsymbol{P}}_{i j}$ and $\overline{\boldsymbol{P}}_{i j} \boldsymbol{S} \boldsymbol{p}_{i j}=\boldsymbol{S} \boldsymbol{p}_{i j}$ (see the proof of Prop. 1 and [10]), and the general formula for expressing the derivative of an eigenvalue w.r.t. a parameter (see, e.g., [22]), it is possible to obtain the following expression for $\lambda_{6}^{B}$ (details are omitted for sake of space)

$\lambda_{6}^{B}=\sum_{(i, j) \in \mathcal{E}}\left(\boldsymbol{v}_{p_{i j}}^{T} \frac{\overline{\boldsymbol{P}}_{i j}}{d_{i j}^{2}} \boldsymbol{v}_{p_{i j}}+2 \boldsymbol{v}_{p_{i j}}^{T} \boldsymbol{S} \frac{\overline{\boldsymbol{p}}_{i j}}{d_{i j}} v_{\psi_{i}}-v_{\psi_{i}}^{2} \overline{\boldsymbol{p}}_{i j}^{T} \boldsymbol{S}^{2} \overline{\boldsymbol{p}}_{i j}\right)$

where $\boldsymbol{v}_{p_{i j}}=\boldsymbol{v}_{p_{i}}-\boldsymbol{v}_{p_{j}}$.

The expression (18) obviously depends on world-frame quantities. An equivalent expression in terms of body-frame quantities can be obtained as follows: let $\boldsymbol{\nu}_{6}$ represent the body-frame normalized eigenvector, i.e., such that $\lambda_{6}^{B}=$ $\boldsymbol{\nu}_{6}^{T} \boldsymbol{B}_{\mathcal{G}} \boldsymbol{\nu}_{6}$. From (15) it follows that $\boldsymbol{v}_{6}=\boldsymbol{T} \boldsymbol{\nu}_{6}$ and, therefore, $\boldsymbol{v}_{p_{i}}=\boldsymbol{R}_{i} \boldsymbol{\nu}_{p_{i}}$ and $v_{\psi_{i}}=\nu_{\psi_{i}}$. Since $\overline{\boldsymbol{P}}_{i j}=\boldsymbol{R}_{i} \boldsymbol{P}_{i j} \boldsymbol{R}_{i}^{T}$ and $\overline{\boldsymbol{p}}_{i j}=\boldsymbol{R}_{i} \boldsymbol{\beta}_{i j}$ (see Prop. 1 and (2)), the expression (18) can be reformulated as

$$
\begin{aligned}
\lambda_{6}^{B} & =\sum_{(i, j) \in \mathcal{E}}\left(\boldsymbol{\nu}_{p_{i j}}^{T} \frac{\boldsymbol{P}_{i j}}{d_{i j}^{2}} \boldsymbol{\nu}_{p_{i j}}+2 \boldsymbol{\nu}_{p_{i j}}^{T} \boldsymbol{S} \frac{\boldsymbol{\beta}_{i j}}{d_{i j}} \nu_{\psi_{i}}-\nu_{\psi_{i}}^{2} \boldsymbol{\beta}_{i j}^{T} \boldsymbol{S}^{2} \boldsymbol{\beta}_{i j}\right) \\
& =\sum_{(i, j) \in \mathcal{E}} l_{i j}
\end{aligned}
$$

with $\boldsymbol{\nu}_{p_{i j}}=\boldsymbol{\nu}_{p_{i}}-{ }^{i} \boldsymbol{R}_{j} \boldsymbol{\nu}_{p_{j}}$. Each term $l_{i j}$ in (19) is now rewritten in terms of only body-frame quantities relative to agents $i$ and $j$ (measured bearings $\boldsymbol{\beta}_{i j}$, interdistances $d_{i j}$, relative orientations ${ }^{i} \boldsymbol{R}_{j}$ and relative components of the body-frame rigidity eigenvector $\boldsymbol{\nu}_{6}$ ). Note that, in general, $l_{i j} \neq l_{j i}$. Furthermore, by looking at the (world-frame) expression of $l_{i j}$ in (18), it follows that $\partial l_{i j} / \partial \psi_{k}=0, \forall k$ (in accordance with Prop. 1).

Let us now introduce the weights $w_{i j}$ into the rigidity eigenvalue $\lambda_{6}^{B}$ and proceed to obtain a closed-form expression for its gradient. As explained at the beginning of Sect. III, the weights $w_{i j}$ can be included by replacing the body-frame symmetric rigidity matrix with a weighted counterpart $\mathcal{B}_{\mathcal{G}}^{T}(\boldsymbol{q}) \boldsymbol{W} \mathcal{B}_{\mathcal{G}}(\boldsymbol{q})$ (and analogously for the worldframe symmetric rigidity matrix). Since $\boldsymbol{W}$ is a diagonal matrix, repeating the previous steps simply results into

$$
\lambda_{6}^{B}=\sum_{(i, j) \in \mathcal{E}} w_{i j} l_{i j}
$$

in place of (19). Expression (20) can be leveraged for obtaining the gradient of $\lambda_{6}^{B}$ w.r.t. the configuration $\boldsymbol{q}_{v}=\left(\boldsymbol{p}_{v}, \psi_{v}\right)$ of a specific agent $v$ in the group. Recalling the definition of set $\mathcal{O}_{i}$ in Sect. II-B, we first note that the only terms $l_{i j}$ that depend on $\boldsymbol{p}_{v}$ are those associated to the edges $(v, j)$, $\forall j \in \mathcal{N}_{v}$ and $(j, v), \forall j \in \mathcal{O}_{v}$. The gradient of these terms w.r.t. $\boldsymbol{p}_{v}$ can be shown (after some (tedious) steps which are, 




Fig. 3: A representative shape for the function $V_{\lambda}\left(\lambda_{6}^{B}\right)$ with $\lambda_{6}^{\min }=0.01$ and $\lambda_{6}^{\max }=0.42$

again, omitted here for the sake of space) to be

$$
\begin{aligned}
\nabla_{\boldsymbol{p}_{v}} l_{v j} & =2 \boldsymbol{R}_{v}\left(\frac{1}{d_{v j}^{3}}\left(\boldsymbol{\beta}_{v j} \boldsymbol{\nu}_{p_{v j}}^{T}+\boldsymbol{\nu}_{p_{v j}}^{T} \boldsymbol{\beta}_{v j} \boldsymbol{I}_{3}\right) \boldsymbol{P}_{v j} \boldsymbol{\nu}_{p_{v j}}+\right. \\
& \left.+\frac{\nu_{\psi_{v}}}{d_{v j}^{2}}\left(\boldsymbol{P}_{v j}-\boldsymbol{\beta}_{v j} \boldsymbol{\beta}_{v j}^{T}\right) \boldsymbol{S} \boldsymbol{\nu}_{p_{v j}}+\frac{\nu_{\psi_{v}}^{2}}{d_{v j}} \boldsymbol{P}_{v j} \boldsymbol{S}^{2} \boldsymbol{\beta}_{v j}\right) \\
& =2 \boldsymbol{R}_{v} \boldsymbol{L}_{v j}
\end{aligned}
$$

and $\nabla_{\boldsymbol{p}_{v}} l_{j v}=-2 \boldsymbol{R}_{j} \boldsymbol{L}_{j v}$.

The gradient of weights $w_{i j}$ is, however, more involved since the weight design introduces additional dependencies which must be correctly taken into account. Consider again the configuration $\boldsymbol{q}_{v}=\left(\boldsymbol{p}_{v}, \psi_{v}\right)$ of a specific agent $v$ and the weight partition (8): clearly, the three subweights $w_{R_{i j}}$ in (9), $w_{F_{i j}}$ in (10) and $w_{V_{i j}}$ in (14) depend on $\boldsymbol{p}_{v}$ over the same set of edges $(v, j), \forall j \in \mathcal{N}_{v}$ and $(j, v), \forall j \in \mathcal{O}_{v}$ as for the terms $l_{i j}$. However, because of its definition, and unlike the terms $l_{i j}$, the third subweight $w_{V_{i j}}$ depends on $\boldsymbol{p}_{v}$ also over all the edges $(j, m), \forall j \in \mathcal{O}_{v}, \forall m \in \mathcal{N}_{j}$. Furthermore, weight $w_{F_{i j}}$ depends on $\psi_{v}$ over all the edges $(v, j), \forall j \in \mathcal{N}_{v}$, while weights $w_{R_{i j}}$ and $w_{V_{i j}}$ do not depend on $\psi_{v}\left(w_{R_{i j}}\right.$ and $w_{V_{i j}}$ are functions of interdistances, and $w_{V_{i j}}$ is also function of the angular quantity $\eta_{i j k}$ which, however, does not depend on $\psi$ ).

Therefore, after some (again tedious) algebra, and by exploiting (21), the gradient of $\lambda_{6}^{B}$ w.r.t. $\boldsymbol{q}_{v}$ can be expanded as follows

$$
\left\{\begin{array}{l}
\nabla_{\boldsymbol{p}_{v}} \lambda_{6}^{B}=\boldsymbol{R}_{v} \sum_{j \in \mathcal{N}_{v}}\left(l_{v j} \nabla_{\boldsymbol{p}_{v}} w_{v j}+2 \boldsymbol{L}_{v j} w_{v j}\right)+ \\
+\sum_{j \in \mathcal{O}_{v}}\left(-2 \boldsymbol{R}_{j} w_{j v} \boldsymbol{L}_{j v}+\sum_{m \in \mathcal{N}_{j}} \boldsymbol{R}_{m} l_{j m} \nabla_{\boldsymbol{p}_{v}} w_{j m}\right) . \\
\nabla_{\psi_{v}} \lambda_{6}^{B}=\sum_{j \in \mathcal{N}_{v}} l_{v j} \nabla_{\psi_{v}} w_{v j}
\end{array}\right.
$$

\section{The bearing rigidity maintenance controller}

Having obtained an explicit expression for the gradient $\nabla_{\boldsymbol{q}_{v}} \lambda_{6}^{B}$, we can now present the proposed bearing rigidity maintenance controller. Similarly to [3], [9], we assume that the agents need to maintain a minimum level of bearing rigidity during motion, i.e., guarantee that $\lambda_{6}^{B}(t)>\lambda_{6}^{\min }>0$ where $\lambda_{6}^{\min }$ is a suitable lower threshold depending on the particular application. We then introduce a potential function $V_{\lambda}\left(\lambda_{6}^{B}\right) \geq 0$ such that $V_{\lambda}\left(\lambda_{6}^{B}\right) \rightarrow \infty$ as $\lambda_{6}^{B} \rightarrow \lambda_{6}^{\min }$ and $V_{\lambda}\left(\lambda_{6}^{B}\right) \rightarrow 0$ (with vanishing derivative) as $\lambda_{6}^{B} \rightarrow \lambda_{6}^{\max }>$ $\lambda_{6}^{\min }$. Fig. 3 shows a possible shape for the potential function $V_{\lambda}\left(\lambda_{6}^{B}\right)$.
Each agent $v$ will then follow the anti-gradient of $V_{\lambda}\left(\lambda_{6}^{B}\right)$ w.r.t. $\boldsymbol{q}_{v}$ which, using (1), yields the following body-frame velocity commands

$$
\left\{\begin{array}{l}
\boldsymbol{u}_{v}=-\boldsymbol{R}_{v}^{T} \nabla_{\boldsymbol{p}_{v}} V_{\lambda}\left(\lambda_{6}^{B}\right) \\
w_{v}=-\nabla_{\psi_{v}} V_{\lambda}\left(\lambda_{6}^{B}\right)
\end{array}\right.
$$

By plugging (22) in (23), we then obtain

$$
\left\{\begin{array}{l}
\boldsymbol{u}_{v}=-\frac{\partial V_{\lambda}}{\partial \lambda_{6}^{B}}\left(\sum_{j \in \mathcal{N}_{v}}\left(l_{v j} \nabla_{\boldsymbol{p}_{v}} w_{v j}+2 \boldsymbol{L}_{v j} w_{v j}\right)+\right. \\
\left.+\sum_{j \in \mathcal{O}_{v}}\left(-2^{v} \boldsymbol{R}_{j} 2 w_{j v} \boldsymbol{L}_{j v}+\sum_{m \in \mathcal{N}_{j}}{ }^{v} \boldsymbol{R}_{m} l_{j m} \nabla_{\boldsymbol{p}_{v}} w_{j m}\right)\right) . \\
w_{v}=-\frac{\partial V_{\lambda}}{\partial \lambda_{6}^{B}} \sum_{j \in \mathcal{N}_{v}} l_{v j} \nabla_{\psi_{v}} w_{v j}
\end{array}\right.
$$

\section{Discussion}

We conclude with some remarks about the bearing rigidity maintenance controller (24). Let us first consider the issue of decentralization and scalability: by analyzing the explicit expressions of the various terms in (24) (given in the previous sections), it is possible to conclude that, in order to implement the rigidity maintenance action, agent $v$ needs knowledge of $\lambda_{6}^{B}$ (for evaluating $\left.\partial V_{\lambda} / \partial \lambda_{6}^{B}\right)$, of $\left(\boldsymbol{\nu}_{p_{v}}, \nu_{\psi_{v}}\right)$, and of

1) $\boldsymbol{\beta}_{v j}, d_{v j}, \psi_{v j}, \nu_{p_{j}}, \nu_{\psi_{j}}, \forall j \in \mathcal{N}_{v}$;

2) $\boldsymbol{\beta}_{j v}, d_{j v}, \psi_{j v}, \nu_{p_{j}}, \nu_{\psi_{j}}, \forall j \in \mathcal{O}_{v}$;

3) $\boldsymbol{\beta}_{j m}, d_{j m}, \psi_{j m}, \nu_{p_{m}}, \nu_{\psi_{m}}, \forall j \in \mathcal{O}_{v}, \forall m \in \mathcal{N}_{j} /\{v\}$.

Let us assume, for now, that each agent has access to the value of $\lambda_{6}^{B}$ and to its own components of $\boldsymbol{\nu}_{6}$ : one can then verify that 1)-3) consist of (measured) bearings, interdistances, relative orientations, and components of the eigenvector $\boldsymbol{\nu}_{6}$, i.e., all quantities available to the robot group. In particular, the quantities in items 1)-2) are either locally available to agent $v$, or can be computed ${ }^{5}$ by exploiting communication with its 1-hop (communication) neighbors $j \in \mathcal{N}_{v}$ (the agents measured by $v$ ) and $j \in \mathcal{O}_{v}$ (the agents measuring $v$ ). The quantities in item 3 ) (which are ultimately due to weights $w_{V_{i j}}$ in (14)) are instead relative to the 1-hop neighbors $m \in \mathcal{N}_{j} /\{v\}$ of any agent $j \in \mathcal{O}_{v}$. Therefore, agent $v$ needs to receive this information from some of its 2-hop communication neighbors. In any case, since the amount of information in 1)-3) is constant per neighbor, an upper bound of the communication complexity for an agent $v$ is $O\left(\left|\mathcal{N}_{v}\right|+\left|\mathcal{O}_{v}\right| \cdot \max _{j \in \mathcal{O}_{v}}\left|\mathcal{N}_{j}\right|\right)$. We can then conclude that the rigidity maintenance controller (24) admits a decentralized implementation by, however, assuming a 2 hop communication model.

A second remark is about the availability, for each agent $v$, of $\lambda_{6}^{B}$ (a global quantity) and of its own eigenvector components $\left(\boldsymbol{\nu}_{p_{v}}, \nu_{\psi_{v}}\right)$ : although these quantities are not directly measurable, it is in principle conceivable to estimate

\footnotetext{
${ }^{5}$ We recall that, as discussed in Sect. II-C, the interdistances $d_{i j}$ and relative orientations $\psi_{i j}$ can be computed by any neighboring pair by exchanging the local estimates $\hat{\boldsymbol{p}}_{i}, \hat{\boldsymbol{p}}_{j}, \hat{\psi}_{i}, \hat{\psi}_{j}$.
} 
them in a decentralized way by adapting the estimators presented in [3], [22] for the connectivity case, and ported in [9] to the (distance) rigidity case. Indeed, these methods essentially require an explicit characterization of the nullspace of the bearing rigidity matrix (which is well understood, see [10]), the use of some PI consensus filters, and the suitable exploitation of the structure of the symmetric bearing rigidity matrix. The estimation of $\lambda_{6}^{B}$ and of $\left(\boldsymbol{\nu}_{p_{v}}, \nu_{\psi_{v}}\right)$ is, however, left for future extensions of this work and in the following results we will just assume availability of these quantities.

A final remark is about the well-posedness of controller (24): as well-known, the derivative of an eigenvalue is not well-defined for multiplicities larger than one (repeated eigenvalues), since in these cases one cannot reliably find/estimate a unique eigenvector to be plugged in the derivative computation [23]. This difficulty, which affects any method based on the optimization of a single eigenvalue associated to some matrix of interest, has already been recognized in [3], [9], [22] without, however, proposing an explicit solution to deal with it. A possible workaround is to replace the eigenvalue to be optimized with a 'smoothed' version [24] which is well-behaved for multiplicities larger than one. Following [17], a possibility is to replace $\lambda_{6}^{B}$ with the quantity $\bar{\lambda}^{B}=\sum_{i=6}^{4 N} \sqrt[p]{\left(\lambda_{i}^{B}\right)^{p}}$ where $\lambda_{i}^{B}$ is the $i$-th eigenvalue of the symmetric bearing rigidity matrix, and $p \ll 0$. Indeed, when $\lambda_{6}^{B} \ll \lambda_{7}^{B}$ one has $\bar{\lambda}^{B} \approx \lambda_{6}^{B}$ while when, instead, $\lambda_{6}^{B} \approx \lambda_{7}^{B} \approx \lambda_{m}^{B} \ll \lambda_{m+1}^{B}$ it is $\bar{\lambda}^{B} \approx \sqrt[p]{m-6} \lambda_{6}^{B}$. Therefore, maximization of $\bar{\lambda}^{B}$ results into maximization of $\lambda_{6}^{B}$ with however the advantage of $\bar{\lambda}^{B}$ being always differentiable. By evaluating $V_{\lambda}(\cdot)$ on $\bar{\lambda}^{B}$, controller (23) then becomes

$$
\left\{\begin{array}{l}
\boldsymbol{u}_{v}=-\frac{\partial V_{\lambda}}{\partial \bar{\lambda}^{B}} \boldsymbol{R}_{v}^{T} \sum_{i=6}^{4 N}\left(\frac{\lambda_{i}^{B}}{\bar{\lambda}^{B}}\right)^{p-1} \nabla_{\boldsymbol{p}_{v}} \lambda_{i}^{B} \\
w_{v}=-\frac{\partial V_{\lambda}}{\partial \bar{\lambda}^{B}} \sum_{i=6}^{4 N}\left(\frac{\lambda_{i}^{B}}{\bar{\lambda}^{B}}\right)^{p-1} \nabla_{\psi_{v}} \lambda_{i}^{B}
\end{array} .\right.
$$

Evaluation of (25) by an agent $v$ would require the same quantities listed in 1)-3) and, in order to evaluate $\partial V_{\lambda} / \partial \bar{\lambda}^{B}$ and $\nabla_{\boldsymbol{q}_{v}} \lambda_{i}^{B}$, the additional availability of $\lambda_{i}^{B}$ and of $\left(\boldsymbol{\nu}_{p_{v}}^{i}, \nu_{\psi_{v}}^{i}\right.$ ) (the $v$-th components of the eigenvector $\boldsymbol{\nu}^{i}$ associated to $\lambda_{i}$ ) for $i=6 \ldots 4 N$. Therefore any implementation of (25) would be decentralized but not scalable, as the amount of information per agent would increase with the size of the whole agent group.

\section{EXPERIMENTAL RESULTS}

This section reports some experimental results meant to validate the proposed bearing maintenance controller (the reader is also referred to the attached video). We employed a group of five quadrotor UAVs MK-Quadro from MikroKopter (see Fig. 4(b)). The usual MK-Quadro setup was extended with an ODROID-XU4 Linux Computer running ROS and the TeleKyb framework [25] for implementing the low-level flight control receiving the body-frame velocity commands (25), and for exchanging data via Wi-Fi with

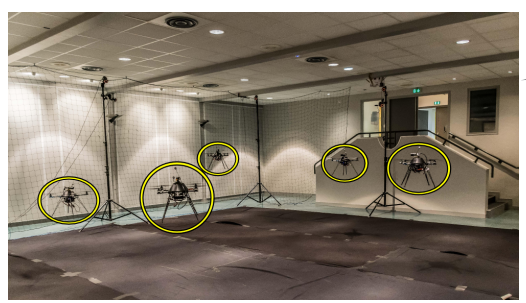

(a)

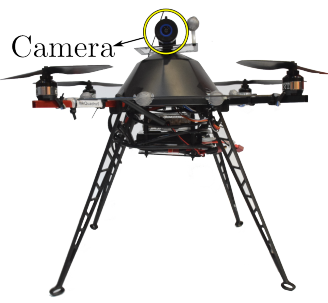

(b)
Fig. 4: The flying arena (a) and our quadrotor platform (b)

the other robots in the group and the ground station. Each quadrotor was also equipped with an onboard camera (a Flea FL3-U3-32S2C-CS by Point-Grey) as shown in Fig. 4(b). The UAVs were flying in an indoor room with a volume of $6.5 \mathrm{~m} \times 5 \mathrm{~m} \times 3 \mathrm{~m}$ and equipped with the Vicon motion capture system (Fig. 4(a)). This was used for obtaining the ground truth and for reconstructing the body-frame bearing measurements $\boldsymbol{\beta}_{i j}$ that would have been obtained by the onboard cameras at $60 \mathrm{~Hz}$. Indeed, we unfortunately found the problem of detecting and tracking (in a reliable way) each quadrotor during flight to be non-trivial, and then exploited the onboard cameras only for the sake of verifying that, during motion, all the neighboring pairs remained indeed well visible in the corresponding camera fov as predicted by the proposed machinery ${ }^{6}$ (see also the attached video).

The experimental results are shown in Figs. 5-6: at the beginning the UAVs just followed the control action (25) and, as a consequence, the value of $\bar{\lambda}^{B}(t)$ was maximized (until the vertical dashed line in Fig. 5a). Then two human operators started acting on two UAVs by sending two velocity commands added to (25) with the aim of triggering as much as possible the loss/gain of neighbors because of the considered sensing constraints. One can then note how presence of these two additional velocity inputs (which are representative of any external planner/algorithm in charge of steering the robot formation in the environment) did not threaten bearing rigidity of the formation thanks to the maintenance action (25) (indeed, as shown in Fig. 5a, $\bar{\lambda}(t)$ remains larger than the chosen threshold $\bar{\lambda}^{\text {min }}=0.01$ ). This is also confirmed in Fig. 6a where the evolution of $\lambda_{6}^{B}(t)$ for the unweighted framework is reported. Finally, Fig. 5b shows the number of edges $|\mathcal{E}|$ over time, thus confirming the (intentionally induced) time-varying nature of the sensing topology during motion, and Fig. 6b reports two of the graph topologies encountered during the experiment.

\section{CONCLUSIONS}

This paper proposed a decentralized gradient-based controller able to enforce bearing rigidity maintenance for a group of quadrotor UAVs while coping with three typical sensing constraints of onboard cameras: minimum/maximum range, limited fov, and possible occluded visibility. The proposed control action exploited a closed-form expression

\footnotetext{
${ }^{6}$ We are currently working towards a fully-onboard implementation of the approach.
} 


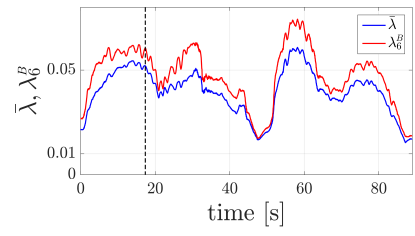

(a)

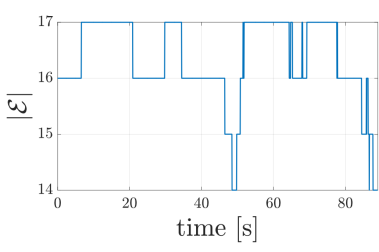

(b)
Fig. 5: Behavior of the $p$-norm $\bar{\lambda}$, of the rigidity eigenvalue $\lambda_{6}^{B}$ (a) and of the number of edges $|\mathcal{E}|$ of the considered framework (b).

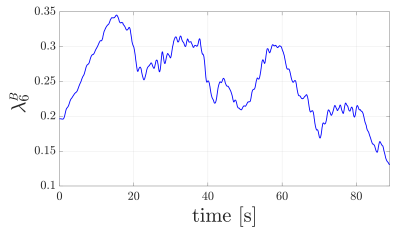

(a)

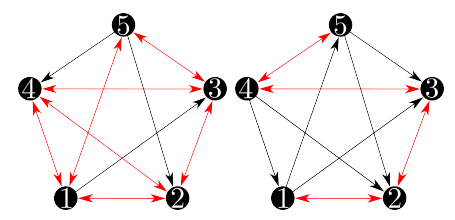

(b)
Fig. 6: Behavior of the unweighted rigidity eigenvalue $\lambda_{6}^{B}$ (a) and two graph topologies corresponding to the maximum (left, $|\mathcal{E}|=$ 17 ) and minimum (right, $|\mathcal{E}|=14$ ) number of edges during the experiment (b).

of $\lambda_{6}^{B}$, the sixth smallest eigenvalue of the symmetric bearing rigidity matrix, and of its gradient w.r.t. the agent configuration. To the best of our knowledge, this is the first work addressing the problem of bearing rigidity maintenance for a formation of UAVs by also explicitly taking into account (complex) sensing constraints. The reported experimental results showed the effectiveness of the proposed control strategy.

Several extensions of this work are possible: first of all, we are currently working towards a fully-onboard implementation by exploiting the onboard cameras for retrieving the bearing measurements w.r.t. neighboring robots. Furthermore, we would like to address the open points listed in Sect. III-D, that is, decentralized estimation of the components of the body-frame eigenvector $\nu_{6}$, and the scalability issue of exploiting the $p$-norm $\bar{\lambda}$ in place of $\lambda_{6}^{B}$. Finally, we are also considering the possibility of extending the proposed weighting machinery for including a collision avoidance action among quadrotors (which is now not guaranteed), similarly to what done in [9].

\section{REFERENCES}

[1] D. Fox, J. Ko, K. Konolige, B. Limketkai, D. Schulz, and B. Stewart, "Distributed multirobot exploration and mapping," Proc. of the IEEE, vol. 94, no. 7, pp. 1325-1339, 2006.

[2] N. Michael, J. Fink, and V. Kumar, "Cooperative manipulation and transportation with aerial robots," in 2009 Robotics: Science and Systems, Seattle, WA, Jun. 2009.

[3] P. Robuffo Giordano, A. Franchi, C. Secchi, and H. H. Bülthoff, "A passivity-based decentralized strategy for generalized connectivity maintenance," The Int. J. of Robotics Research, vol. 32, no. 3, pp. 299-323, 2013.

[4] K. Hausman, J. Müller, A. Hariharan, N. Ayanian, and G. S. Sukhatme, "Cooperative multi-robot control for target tracking with onboard sensing," IEEE Trans. on Robotics, vol. 34, no. 13, pp. 1660-1677, 2015 .
[5] E. Montijano, E. Cristofalo, D. Zhou, M. Schwager, and C. Sagues, "Vision-based distributed formation control without an external positioning system," IEEE Trans. on Robotics, vol. 32, no. 2, pp. 339-351, Jul. 2016.

[6] B. D. O. Anderson, Y. Changbin, B. Fidan, and J. Hendrickx, "Rigid graph control architectures for autonomous formations," IEEE Control Systems Magazine, vol. 6, no. 28, pp. 48-63, 2008.

[7] L. Krick, M. E. Broucke, and B. A. Francis, "Stabilisation of infinitesimally rigid formations of multi-robot networks," Int. J. of Control, vol. 82, no. 3, pp. 423-439, Mar. 2009.

[8] T. Eren, "Formation shape control based on bearing rigidity," Int. J. of Control, vol. 9, no. 85, pp. 1361-1379, 2012.

[9] D. Zelazo, A. Franchi, H. H. Bülthoff, and P. Robuffo Giordano, "Decentralized Rigidity Maintenance Control with Range-only Measurements for Multi-Robot Systems," Int. J. of Robotics Research, vol. 34, no. 1, pp. 105-128, 2015.

[10] F. Schiano, A. Franchi, D. Zelazo, and P. Robuffo Giordano, "A rigidity-based decentralized bearing formation controller for groups of quadrotor UAVs," in 2016 IEEE/RSJ International Conference on Intelligent Robots and Systems (IROS 2016), 2016.

[11] D. Zelazo, P. Robuffo Giordano, and A. Franchi, "Bearing-Only Formation Control Using an SE(2) Rigidity Theory," in 2015 IEEE $C D C$, Osaka, Japan, Dec. 2015, pp. 6121-6126.

[12] S. Zhao and D. Zelazo, "Bearing rigidity and almost global bearingonly formation stabilization," IEEE Transactions on Automatic Control, vol. 61, no. 5, pp. 1255-1268, 2016.

[13] J. Aspnes, T. Eren, D. K. Goldenberg, A. S. Morse, W. Whiteley, Y. R. Yang, B. D. O. Anderson, and P. N. Belhumeur, "A theory of network localization," IEEE Trans. on Mobile Computing, vol. 5, no. 12, pp. 1663-1678, 2006.

[14] D. Zelazo, A. Franchi, and P. Robuffo Giordano, "Rigidity Theory in $S E(2)$ for Unscaled Position Estimation using only Bearing Measurements," in 2014 ECC, Strasbourg, France, Jun. 2014, pp. 2703-2708.

[15] I. Shames, A. N. Bishop, and B. D. O. Anderson, "Analysis of Noisy Bearing-Only Network Localization," IEEE Trans. on Automatic Control, vol. 58, no. 1, pp. 247-252, Jan. 2013.

[16] R. Tron, L. Carlone, F. Dellaert, and K. Daniilidis, "Rigid components identification and rigidity enforcement in bearing-only localization using the graph cycle basis," in 2015 ACC, Chicago, IL, Jul. 2015, pp. 3911-3918.

[17] R. Spica and P. Robuffo Giordano, "Active Decentralized Scale Estimation for Bearing Based Localization," in 2016 IEEE IROS, Daejeon, Korea, Oct. 2016.

[18] A. Franchi, C. Masone, V. Grabe, M. Ryll, H. H. Bülthoff, and P. Robuffo Giordano, "Modeling and control of UAV bearingformations with bilateral high-level steering," The Int. J. of Robotics Research, Special Issue on 3D Exploration, Mapping, and Surveillance, vol. 31, no. 12, pp. 1504-1525, 2012.

[19] I. Shames, A. N. Bishop, and B. D. O. Anderson, "Analysis of noisy bearing-only network localization," IEEE Trans. on Automatic Control, vol. 1, no. 58, pp. 247-252, 2013.

[20] A. Cornejo, A. J. Lynch, E. Fudge, S. Bilstein, M. Khabbazian, and J. McLurkin, "Scale-free coordinates for multi-robot systems with bearing-only sensors," The Int. J. of Robotics Research, vol. 12, no. 32, pp. 1459-1474, 2013.

[21] S. Zhao and D. Zelazo, "Bearing-Based Distributed Control and Estimation of Multi-Agent Systems," in European Control Conference, Linz, Austria, 2015, pp. 2207-2212.

[22] P. Yang, R. A. Freeman, G. J. Gordon, K. M. Lynch, S. S. Srinivasa, and R. Sukthankar, "Decentralized estimation and control of graph connectivity for mobile sensor networks," Automatica, vol. 46, no. 2 , pp. 390-396, 2010 .

[23] M. I. Friswell, "The derivatives of repeated eigenvalues and their associated eigenvectors," Journal of Vibration and Acoustics, vol. 118 , pp. 390-397, 1996.

[24] X. Chen, H. Qi, L. Qi, and K.-L. Teo, "Smooth convex approximation to the maximum eigenvalue function," Journal of Global Optimization, vol. 30, no. 2, pp. 253-270, 2004.

[25] V. Grabe, M. Riedel, H. H. Bulthoff, P. Robuffo Giordano, and A. Franchi, "The telekyb framework for a modular and extendible ros-based quadrotor control," in 2013 IEEE ECMR, Barcelona, Spain, Sep. 2013, pp. 19-25. 